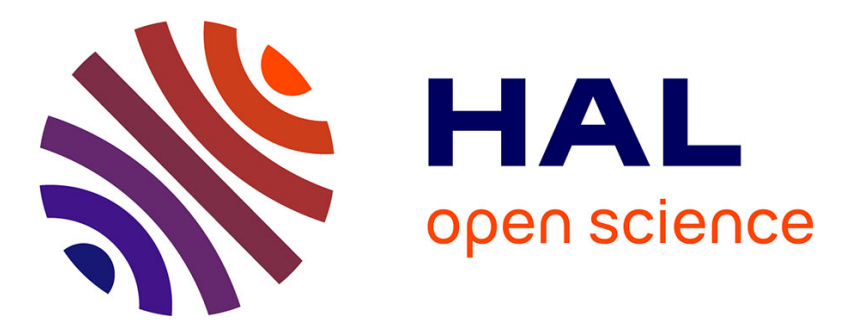

\title{
Putative protective effect of inspiratory threshold loading against exercise-induced supraspinal diaphragm fatigue
}

Sophie Antoine-Jonville, Luce Jutand, Thomas Similowski, André Denjean, Nathalie Delpech

\section{To cite this version:}

Sophie Antoine-Jonville, Luce Jutand, Thomas Similowski, André Denjean, Nathalie Delpech. Putative protective effect of inspiratory threshold loading against exercise-induced supraspinal diaphragm fatigue. Journal of Applied Physiology, 2005, 98 (3), pp.991-998. 10.1152/japplphysiol.00528.2004 . hal-01203652

\section{HAL Id: hal-01203652 \\ https://hal.science/hal-01203652}

Submitted on 27 Mar 2017

HAL is a multi-disciplinary open access archive for the deposit and dissemination of scientific research documents, whether they are published or not. The documents may come from teaching and research institutions in France or abroad, or from public or private research centers.
L'archive ouverte pluridisciplinaire HAL, est destinée au dépôt et à la diffusion de documents scientifiques de niveau recherche, publiés ou non, émanant des établissements d'enseignement et de recherche français ou étrangers, des laboratoires publics ou privés.

\section{(c) (1) $\$$}

Distributed under a Creative Commons Attribution - NonCommercial| 4.0 International 


\section{Putative protective effect of inspiratory threshold loading against exercise-induced supraspinal diaphragm fatigue}

RUNNING HEAD: EXERCISE-INDUCED DIAPHRAGM SUPRASPINAL FATIGUE

KEYWORDS: FATIGUE - RESPIRATORY MUSCLES - TRANSCRANIAL MAGNETIC

STIMULATION - MOTOR CORTEX

S. Jonville $\mathbb{1}^{1}$, L. Jutand ${ }^{1}$, T. Similowski ${ }^{2}$, A. Denjean ${ }^{1,3}$, and N. Delpech ${ }^{1}$

1. Laboratoire des Adaptations Physiologiques aux Activités Physiques, UPRES EA 3813, Faculté des Sciences du Sport, 4 allée Jean Monnet, 86000 Poitiers, France

2. Laboratoire de Physiopathologie Respiratoire, Service de Pneumologie, Groupe Hospitalier Pitié-Salpêtrière, 47-83 Bd de l'Hôpital, 75651 Paris Cedex 13, France \& UPRES EA 2397, Université Paris VI Pierre et Marie Curie, Paris, France

3. Service d'Explorations Fonctionnelles, Physiologie Respiratoire et de l'Exercice. Pavillon Beauchant, CHU de Poitiers, B.P. 577, 86000 Poitiers, France

Correspondence: Dr. Sophie Jonville

E-mail: sophie.jonville@wanadoo.fr

Tel: (+ 590) 590489227

Fax: (+ 590) 590499279 
The present investigation was intended to assess the consequences of an inspiratory load on the diaphragm central component of fatigue during exercise. We recorded the motor potential (MEP) evoked by transcranial magnetic stimulation of the motor cortex in ten subjects. The diaphragm and rectus femoris were studied before and 10, 20 and 40 min after two 16 -min cycling exercise trials requiring $55 \%$ of $\mathrm{V}_{2 \mathrm{MAx}}$ i) one with an inspiratory threshold load (E + ITL) corresponding to $10 \%$ of maximal inspiratory pressure and ii) the other without the load (E). Dyspnea, heart rate, electromyographic activity of the sternocleidomastoid, and diaphragm work were significantly higher in E + ITL than in E. Neither trial affected the response to phrenic magnetic stimulation, which was performed 15 and 25 minutes post-exercise, or the maximal inspiratory pressure $\left(116 \mathrm{cmH}_{2} \mathrm{O}\right.$ and 120 $\mathrm{cmH}_{2} \mathrm{O}$ before $\mathrm{E}$ and $\mathrm{E}+\mathrm{ITL}$, respectively, and $110 \mathrm{cmH}_{2} \mathrm{O}$ and $114 \mathrm{cmH}_{2} \mathrm{O}$ at 30 minutes post-exercise). Whereas the amplitude of the diaphragm MEP was unaffected by E + ITL (+ $2.1 \pm 29.4 \%$ ), a significant decrease was observed 10 minutes after $\mathrm{E}$ as compared to baseline $(-37.1 \pm 22.3 \%)$ and as compared to E + ITL. The MEP amplitude of rectus femoris remained unchanged with $\mathrm{E}$ and $\mathrm{E}+\mathrm{ITL}$. The recruitment of synergistic agonists during $\mathrm{E}+$ ITL may have normalized the major ventilatory stress and reset up the excitability of the diaphragm pathway. 


\section{INTRODUCTION}

Fatigue develops when striated skeletal muscles are confronted with loads that challenge their strength and endurance, involving both peripheral (downstream the neuromuscular junction) and central mechanisms (see 13 for a review). Central fatigue is common in humans $(7,8,33)$ and is viewed by some as a mechanism to protect against exercise-induced muscle damage or exercise-induced homeostatic failure (13).

As with other skeletal muscles, inspiratory muscles are susceptible to fatigue both in response to specific loading (16) and after exhaustive endurance exercise. For example, highly fit normal volunteers exhibit decreased static inspiratory pressures after prolonged or extreme whole body exercise [e.g. a triathlon (17) or marathon (20)]. Because of the volitional nature of static inspiratory pressure manoeuvres, the question of the central or peripheral nature of the mechanisms involved has been left open. Johnson et al. (19) found convincing evidence of contractile alterations (peripheral fatigue) when they compared the results of bilateral supramaximal phrenic nerve stimulation before and after exercise-induced diaphragm fatigue in healthy humans. This does not, however, exclude a central component. Indeed, Verin et al. (35) demonstrated supraspinal diaphragm fatigue following incremental treadmill exercise. In this study, the depression of the diaphragmatic motor potentials evoked by transcranial magnetic stimulation was more pronounced and slower to recover than that of the quadriceps.

During $\mathrm{CO}_{2}$-stimulated ventilation (38) or exercise, plastic changes in respiratory muscle recruitment occur. The contribution of the diaphragm tends to decrease, with the muscle acting increasingly as a flow generator rather than a pressure generator (2). The contribution of extradiaphragm inspiratory and expiratory muscles to ventilation augments so as to maintain the global efficacy of the ventilatory pump. This is true when ventilatory loading starts with fresh muscles (19) and is even more evident when the diaphragm has been previously fatigued (30). These adaptive strategies are central in nature and may have a 
protective function for the diaphragm. The present study was designed to address a part of this issue in healthy volunteers. The specific aims were to determine i) whether moderate cycling exercise leads to a depression in motor evoked potentials and ii) how an imposed inspiratory threshold load [known to provoke a strong recruitment of extradiaphragm inspiratory muscles (39)] would influence this phenomenon. We hypothesized that the diaphragm responses to transcranial magnetic stimulation after exercise ewould be dependent on whether the exercise was performed with or without inspiratory threshold loading.

\section{METHODS}

\section{Participants}

Ten healthy volunteers ( 5 men, 5 women; age 21 to 33 yr; body mass index 18.7 to 25.8 kg. $\mathrm{m}^{-2}$; all but one right-handed), free of any disease or significant medical history, were studied. Their maximal oxygen uptake ( $\left(\mathrm{V}_{2 \mathrm{MAX}}\right.$ ) ranged from 32.0 to $56.9 \mathrm{ml} . \mathrm{min}^{-1} \cdot \mathrm{kg}^{-1}$ (see Table 1). They were instructed to maintain their usual level of physical activity during the study period and not to modify their caffeine or nicotine consumption. They refrained from alcohol consumption, sedative intake, and heavy exercise during the 24 hours preceding the tests. Of note, an $11^{\text {th }}$ subject who had been recruited dropped out of the study because he could not tolerate transcranial magnetic stimulation.

Table 1

The protocol had been approved by the appropriate ethics committee and all subjects gave their informed written consent for the study.

\section{Preliminary evaluation}

At the first visit, each subject underwent a physical examination and a 12-lead electrocardiogram (ECG). Maximal oxygen uptake was measured using an electronically braked cycle ergometer (Ergometrics 800, Ergoline, Germany) as follows. After an 8-minute 
warm-up at a heart rate of about 110 beats per minute, the load was increased every minute by 25 watts until the subject was unable to continue despite vigorous coaching. During this procedure, the subjects breathed through a face mask (Hans Rudolph, Kansas City, MO, USA) connected to a pneumotachograph (Type 3, Hans Rudolph, Kansas City, MO, USA). Inspiratory and expiratory gas composition and flows were analyzed breath-by-breath using an automated system (Medisoft, Exp'air 1.26, Belgium). The metabolic and ventilatory parameters, including oxygen uptake $\left(\mathrm{V}_{2}\right)$, carbon dioxide production $\left(\mathrm{V}^{\circ} \mathrm{CO}_{2}\right)$, expiratory flow ( $\mathrm{V} E)$, tidal volume $\left(\mathrm{V}_{\mathrm{T}}\right)$, respiratory rate $(\mathrm{RR})$ and duty cycle (ratio between inspiratory time and total time of the cycle), were monitored continuously and averaged over every $15-\mathrm{s}$ period. Heart rate was recorded (Archimed) continuously by four ECG leads.

The following criteria had to be met to accept the highest $\mathrm{V}_{2}$ mean over $15 \mathrm{~s}$ at the time of task failure as maximal: i) stabilization of $\mathrm{v}_{2}$, ii) a respiratory exchange ratio $>1.1$, and iii) attainment of the age-predicted maximal heart rate (220-age).

The capacity of the subjects to produce static inspiratory pressures was measured in terms of the volitional maximal inspiratory pressure developed from residual volume against an occluded airway ( $\left.\mathrm{PI}_{\mathrm{MAX}}\right)$. Maximal inspiratory pressure was determined as the highest pressure maintained over $1 \mathrm{~s}$ against an occlusion. Mouth pressure was measured by a differential pressure transducer $\left( \pm 250 \mathrm{cmH}_{2} \mathrm{O}\right.$, Validyne, Northridge, CA, USA), digitized at 500 Hz (MP100 Manager V3.2.6, Biopac Systems, Inc., Santa Barbara, CA, USA) and stored on a computer.

\section{Exercise protocol}

Each subject performed two exercise (E) trials, one with an inspiratory threshold load (ITL) added (E + ITL) and one without (E). The runs were performed in random order, at a minimal interval of two days and at the same time of the day. Each trial consisted of a 5- 
minute warm-up at $50 \%$ of the exercising load, immediately followed by a 16 -minute cycling bout. The exercising load required $55 \%$ of $\mathrm{VO}_{2 \mathrm{MAX}}$ and was below the ventilatory anaerobic threshold determined according to Wasserman (37). Subjects were instructed to cycle between 60 and $70 \mathrm{rpm}$. Throughout the tests, gases were analyzed breath-by-breath using the automated system described above.

Inspiratory loading was achieved during the E + ITL runs by connecting an inspiratory threshold valve (threshold IMT, Respironics, Germany) to the inspiratory side of a two-way breathing valve (type 2700, Hans Rudolph, Kansas City, MO, USA) connected to the face mask. The valve was adjusted to open at an inspiratory pressure of about $10 \%$ of $\mathrm{PI}_{\mathrm{MAX}}$. The inspiratory load was added 1 minute after the beginning of exercise. Expiration remained free throughout testing. Mouth pressure and flow were continuously recorded during both trials from the set-up of the inspiratory load.

While exercising, the subjects were asked to rate their dyspnea every $90 \mathrm{~s}$ on a visual scale.

No instructions were given to the subjects regarding breathing pattern.

\section{Muscle activity during exercise}

The electromyographic (EMG) activity of the sternocleidomastoid muscles (SCM) and transdiaphragmatic pressures was monitored continuously during the two trials (E and $\mathrm{E}+$ ITL) on the six subjects numbered 5 to 10 in Table 1.

Spontaneous electromyographic activity of the SCM was obtained by surface recording using pairs of disposable silver cup electrodes. These were placed over the mid-point of the muscle belly (4). The raw EMG signals were full-wave rectified and integrated with time of burst to provide an integrated EMG activity index $\left(\mathrm{EMG}_{\mathrm{i}}\right)$. During the 16-minute cycling bout, the subjects were asked to maintain their posture on the ergocycle and any part of the signal containing interference caused by head movement was removed. 
The transdiaphragmatic pressure was determined as the difference between gastric and oesophageal pressures continuously recorded over the two exercise trials. The catheter tip pressure transducer (CTO-2, Gaeltec Ltd, Scotland, UK) was gently positioned in the oesophagus and stomach, after local anaesthesia of the nasal passage with xylocaine spray (xylocaine 5\% Nebuliser, Astra Inc., France). Transdiaphragmatic pressure deflections were integrated over time $\left(\mathrm{Pdi}_{\mathrm{i}}\right)$ and served to reflect the work of the diaphragm.

\section{Transcranial magnetic stimulation}

The responses to transcranial magnetic stimulation were studied in all subjects, who were seated comfortably in a quiet room, in a relaxed state.

Surface recordings of the diaphragm and rectus femoris electromyograms were obtained using pairs of disposable silver cup electrodes. The right costal diaphragmatic activity was recorded with active electrodes taped to the skin along the mid-clavicular line in the eighth intercostal space (34). For the rectus femoris, the electrodes were placed in the physiological axis over the belly of the muscle (25).

Transcranial magnetic stimulation was performed using a Magstim ${ }^{\circledR} 200$ magnetic stimulator (The Magstim Company, Sheffield, UK) equipped with a conical butterfly coil (coil diameter $110 \mathrm{~mm}$; maximal output $1.6 \mathrm{~T}$ ). The optimal stimulation site and coil position were determined by testing several combinations at the lowest stimulation intensity evoking a response from both the rectus femoris and the diaphragm. To ensure constant body and coil positions, the shadow of the subject as projected from direct lateral lighting and the shadow of the coil in stimulation position were drawn on a board at the beginning of the first session and then carefully reproduced. Care was taken to perform transcranial magnetic stimulation with the diaphragm and the rectus femoris relaxed to avoid any modification in the response by facilitation, and at end-expiration (from visual inspection of rib cage movement). 
A 10-minute rest period preceded the baseline measurements. At rest and 10, 20 and 40 minutes after the end of exercise, sets of five transcranial magnetic stimulations were performed at $100 \%$ of stimulator output. The time interval between stimulations was $30 \mathrm{~s}$.

The electromyographic signals were digitized at $20,000 \mathrm{~Hz}$ for $260 \mathrm{~ms}$ after the stimulation (MP100 Manager V3.2.6, Biopac Systems, Inc., Santa Barbara, CA, USA), analyzed and then saved to a desktop computer. The MEP latency was measured as the time elapsed from the stimulus to the first departure of the electromyographic signal from baseline. Its amplitude was determined as the peak-to-peak difference of the first recognizable deflection.

\section{Cervical magnetic stimulation and maximal inspiratory pressure}

The six subjects in whom muscle activity was studied had a slightly modified exercise protocol: diaphragm responses to phrenic nerve stimulation were also recorded before exercise (after transcranial stimulations) and 15 and 25 minutes after exercise. The purpose of these measurements was to assess any peripheral fatigue that may have developed. These subjects also performed the $\mathrm{PI}_{\mathrm{MAX}}$ manoeuvres described above (see preliminary evaluation) at rest and 30 minutes after the end of each exercise trial.

The phrenic nerves were bilaterally stimulated with cervical magnetic stimulation using $100 \%$ of the maximal output of a Magstim ${ }^{\circledR} 200$ system, and a circular $90-\mathrm{mm}$ coil (S/N, maximum output $2 \mathrm{~T}$, pulse duration $0.05 \mathrm{~ms}$; The Magstim Company, Sheffield, UK). Subjects were seated with the neck slightly flexed; they wore a noseclip and breathed through a mouthpiece with a small leak (to avoid glottis closure). Five stimulations were delivered at the functional residual capacity determined by visual control of chest movements, with at least $30 \mathrm{~s}$ intervals to avoid a twitch on twitch potentiation.

Mouth pressure measurement in response to phrenic nerve stimulation was performed as described in the Preliminary evaluation section. The amplitude of the twitch mouth pressure (twPmo) signal was measured as the difference between baseline and the peak pressure during 
phrenic stimulation. The electromyographic (M-Wave) and mechanical responses to cervical magnetic stimulation were acquired similarly to the responses to transcranial magnetic stimulation (see above). We took particular care in verifying the absence of difference in the M-wave amplitude of the diaphragm.

\section{Statistical procedures}

The distributions of the amplitudes and latencies of the motor evoked potentials were checked for normality using the D test of Kolmogorov-Smirnov. Because none of the MEP distributions were normal, a log-transformation was applied. The normality of the distributions after transformation was confirmed by $\mathrm{D}$ values of 0.077 for the diaphragm in the $\mathrm{E}$ and $\mathrm{E}+\mathrm{ITL}$ trials, and D values of 0.087 and 0.077 for the quadriceps in the $\mathrm{E}$ and $\mathrm{E}+$ ITL trials, respectively (all $p>0.2$ ). The latencies of the diaphragm motor evoked potentials were normally distributed during free and loaded exercise (all $\mathrm{D}<0.1$ and $p>0.2$ ).

Two-way analysis of variance with repeated measures was used to determine differences in the mean values of MEP amplitudes and latencies over the duration of exercise and recovery (Statistica Kernel V 5.5 Statsoft, France). The analysis for MEP amplitudes was performed on log-transformed data.

When a main statistical effect was found by ANOVA, means were compared using a Newman-Keuls post hoc test.

Paired $t$ tests were used to assess differences beween trials (with and without inspiratory load) when the effect of time was not analyzed.

Data are expressed as means \pm SD unless otherwise stated. Differences were considered statistically significant when the probability $p$ of a type I error was 0.05 or less.

\section{RESULTS}




\section{Cardioventilatory data}

Two subjects had to interrupt the loaded exercise trial before its scheduled end because of excessive hyperventilation inducing acute dizziness in one case and intolerable dyspnea in the other. As they stopped only 1 and 2 minutes before the end of exercise, respectively, they were maintained in the analyses.

The mean minute ventilation, breathing frequency, tidal volume and duty cycle recorded over 15 minutes of the exercise were not different between the free and loaded runs (Figure

Fig. 1 1), as reflected by $p$ values of $0.94,0.69,0.13$, and 0.27 , respectively. However, the individual breathing patterns in response to the threshold load varied among the subjects. Overall, the mean inspiratory flow rate $\left(\mathrm{V}_{\mathrm{T}} / \mathrm{Ti}\right)$, which is assumed to reflect the ventilatory command, did not differ between the two runs.

Fig. 2 All the subjects but one scored a significantly higher dyspnea during the trial with inspiratory loading than during the free trial of whole-body exercise (Figure 2A).

The mean heart rate was significantly higher during the loaded run than the free run $(147 \pm$ 15 vs. $144 \pm 18$ bpm, $p=0.03$ ) (Figure 2B).

\section{Muscle activity}

The sternocleidomastoid activity was significantly higher $(5.3 \pm 2.5$ vs. $15.4 \pm 10.4)$ when the inspiratory load was added (Figure 3A, Figure 4). Indeed, the clinical observation of rib cage dynamics supported these results.

Fig. 3 In the only three subjects whose diaphragm work could be measured during the two exercise runs, $\mathrm{Pdi}_{\mathrm{i}}$ increased from $18.3 \pm 6.1 \mathrm{cmH}_{2} \mathrm{O} \cdot \mathrm{ms}^{-1}$ in the free trial to $56.7 \pm 9.0$

Fig. 4 
$\mathrm{cmH}_{2} \mathrm{O} \cdot \mathrm{ms}^{-1}$ in the loaded one, and mean Pdi followed the same increment pattern (Figure 3B, Figure 4).

\section{Cervical magnetic stimulation and maximal inspiratory pressure}

The variance analysis showed that the maximal inspiratory pressure was not affected by the trial (exercise vs. loaded exercise), the effort (after vs. before, whether including an inspiratory load or not) or a combination of these factors (Figure 5A). Similarly, twitch mouth pressures did not change across conditions of time or exercise (Figure 5B). The M-

Fig. 5 wave amplitude also remained unaffected by these factors (Figure 6).

Fig. 6

\section{Responses to transcranial magnetic stimulation}

ANOVA revealed an overall interaction effect between time of measurement and trial condition on the amplitudes of the diaphragm MEP $(p=0.038)$ The amplitudes were not significantly different between the two trials at rest $(p=0.209)$. Ten minutes after the end of $\mathrm{E}$, however, they were significantly lower than at baseline and at the same point in time after E + ITL (Figure 7A); 20 and 40 minutes after E, the amplitudes remained lower than at baseline, although not significantly so ( $p=0.061$ and 0.075 , respectively). Conversely, E + ITL had no effect on the amplitudes of the diaphragm MEP (after $v s$. before, all $p$ values $>0.88$ ).

The average diaphragm MEP latencies were $15.5 \pm 1.1 \mathrm{~ms}$ at baseline in the free condition and $16.0 \pm 1.1 \mathrm{~ms}$ in the loaded condition. The latencies were unchanged over time during a given session, and unaffected by ITL $(p=0.77)$.

Fig. 7 Figure 7B describes the evolution in the amplitudes of the rectus femoris motor evoked potentials with time. The MEP amplitudes were unchanged over time after E, and unaffected 
by ITL. The MEP latencies also remained unchanged between and during the trials $(p=0.98)$. The average latency at rest was $22.1 \pm 2.5 \mathrm{~ms}$ for E and $22.4 \pm 2.1 \mathrm{~ms}$ for $\mathrm{E}+\mathrm{ITL}$.

\section{DISCUSSION}

This study shows that non-exhaustive whole body exercise in normal individuals can induce neurophysiological changes compatible with supraspinal diaphragm fatigue, and that this effect is not present when the exercise is combined with a moderate level of inspiratory threshold loading.

\section{Methodological issues}

A decrease in the amplitude of the motor potentials evoked by transcranial magnetic stimulation can be interpreted in terms of supraspinal fatigue when no significant metrological changes occur. In the present study, we took particular care to maintain a constant body posture and a constant relationship between the stimulating coil and the scalp throughout the experiments (see Methods). In addition, because changes in the inter-electrode impedance or the spatial relationship between the electrodes and the recorded motor units could theoretically have played a role in the variations that we observed, we compared the amplitude of the responses to peripheral stimulation before and after exercise. We observed no variation in the electromyographic response to CMS, which was expected, given that this signal is generally unaffected by fatigue protocols involving non-isotonic efforts. We are thus confident that changes in the stimulus or changes in the recording conditions did not play a significant role in the phenomena that we observed.

The measurement of the pressure generated in response to CMS allowed for the control of peripheral muscle fatigue in this study. In terms of exercise intensity, ventilatory demand, and degree of loading, the experimental conditions were such that contractile fatigue was extremely unlikely to occur, and particularly so in the diaphragm (19). Indeed, the absence of variation in the twitch mouth pressure suggests that peripheral fatigue did not develop. 
Finally, non-fatiguing efforts tend to potentiate the electromyographic response to transcranial magnetic stimulation (3). This "post-exercise facilitation" phenomenon is the result of a transient increase in the excitability of the alpha-motoneurons induced by muscular activity (28), a feature that we did not observe after either type of exercise run. The motor evoked potential latencies in our subjects, which were in the normal range for unfacilitated responses (29), did not differ from their baseline values after exercise. The maximal inspiratory manoeuvre has potential facilitatory effects but it was followed by 10 minutes of rest. The subjects remained quiet, i.e. motionless and silent, during the recovery. Post-exercise facilitation therefore does not seem likely to have been a confounding factor.

\section{Central diaphragm fatigue in response to unloaded exercise}

The twitch interpolation technique has been used to assess the degree of voluntary activation of a given muscle during a voluntary contraction $(24,21)$. It consists of superimposing supramaximal stimulations on the nerve commanding an already contracting muscle. When task failure is associated with an additional mechanical output in response to this stimulation, fatigue is assigned to a central component. Central diaphragmatic fatigue has been evidenced using this technique in various contexts $(6,21)$ characterised by diaphragmoriented loading protocols. Verin et al. (35) seem to have been the first to provide arguments for central diaphragm fatigue after whole-body exercise. They found that both the diaphragm and the quadriceps exhibited post-exercise decrements in their responses to transcranial magnetic stimulation after an incremental walking exercise likely to require high ventilatory outputs. As compared to their study, we did not observe signs of supraspinal fatigue in the recti femori. The reason for this difference may lie in the nature of the exercise paradigm used: our protocol was specifically designed not to provoke exhaustion. The two studies are, however, consistent in that they suggest a greater susceptibility to supraspinal fatigue in the diaphragm than in the quadriceps. This result is compatible with the view that central fatigue has a protective function (13). It is also tempting to draw a parallel between the results of 
these two studies and the fact that normal subjects achieve greater activation of the quadriceps than of the diaphragm during similar loading protocols (14). This feature has been called on by Guleria et al. (14) to explain the relative difficulty of experimentally producing diaphragm contractile fatigue in comparison with other muscles.

Of note, most works on supraspinal fatigue have involved intense muscular efforts, generally mono-articular and leading to task failure. The muscles under study have generally been small and distal $(9,28)$, with some exceptions $(27,33)$. This was not the case for the diaphragm in our experiments, which makes comparison with other results difficult. However, supraspinal fatigue has been observed in several muscles as a result of whole body exercise (18) (as opposed to specific limb solicitation).

\section{Effects of inspiratory threshold loading on the post-exercise diaphragm response}

Increased ventilatory demand is often associated with changes in the activity and recruitment pattern of the respiratory muscle groups $(19,26)$. These changes generally tend to put the burden of the ventilatory load on extradiaphragm muscles. For example, exercise is normally associated with a precocious and strong recruitment of abdominal muscles that directly contribute to the ventilatory work by their expiratory action. They also indirectly help inspiration by bringing the rib cage at end-expiration below its equilibrium position, therefore making rib cage elastic recoil available for inspiration (10). Rib cage and neck muscles are also recruited during exercise. They contribute directly to the expansion of the rib cage, and decrease rib cage distortability (21). As a result, it has been shown that the nature of the diaphragm contraction changes during exercise (2) and therefore its energy expenditure is not increased disproportionately. Studies, particularly on limb muscles, have clearly demonstrated that a given task can be accomplished with different recruitment strategies. For example, fatigue of the vastus lateralis has been shown to modify the recruitment of synergistic muscles, thereby preserving the success of a knee extension task (1). Given the compensatory 
mechanisms that are developed in patients with diaphragm paralysis (15) and in healthy subjects exercising with fatigued inspiratory muscles (30), it also appears reasonable to assume that changes in the pattern of extradiaphragm respiratory muscle activity would allow the ventilatory demand to be met in spite of reduced diaphragm activity. To support this, Johnson et al. (19) showed that twitch transdiaphragmatic pressure was reduced at all lung volumes after task failure in 12 healthy volunteers with a variety of fitness levels exercising to exhaustion at 85 and $95 \%$ of their maximum oxygen uptake. In this study, the contribution of the diaphragm to the respiratory motor output tended to decrease with the duration of the effort, with a shift of the load toward other muscles.

Inspiratory loading favours the recruitment of inspiratory neck and rib cage muscles (30), and the amplitude partly determines endurance. For this study, the choice of $10 \%$ of the maximal inspiratory pressure was based on preliminary studies that showed the load to be demanding and capable of generating strong neck and rib cage muscle contractions. The literature $(5,35)$ reveals that this load is associated with a normal respiratory duty cycle and is unlikely to induce low frequency diaphragm fatigue, which had to be avoided in this study. The recruitment of additional muscles with loading is observed in patients with chronic obstructive pulmonary disease and in healthy subjects. Both resistive (16) and threshold (11) loading lead to strong solicitation and sometimes fatigue $(16,39)$ of extradiaphragmatic muscles. The ITL added to the burden of the exercise-related increase in ventilatory demand consistently resulted in recruitment of extradiaphragmatic muscles, as shown by the electromyographic recording of the sternocleidomastoid muscles. Moreover, our subjects reported dyspnea during the loaded protocol, whereas this was not the case during the "free" protocol. Yet Fitting et al. (12) and Ward et al. (36) both clearly showed that recruiting the sternocleidomastoid and parasternal intercostal muscles rather than the diaphragm to perform a given respiratory task is strongly associated with dyspneic sensations. Therefore, we suggest 
that the redistribution in the respective roles of the inspiratory muscles is related to the disappearance of the post-exercise depression in diaphragm MEPs in the E + ITL condition.

This is not a simple assertion that the decrease in motor evoked potentials is due to lower recruitment of the diaphragm. There is probably no correlation between the amount of work produced by a muscle group at a given time and the fatigue of its command. Instead, we propose that the decreased excitability after exercise is linked to the reorganization of the diaphragm's motor command. When moderate exercise is not prolonged, this reorganization could optimize the use of resources. Changing the breathing has already been presented as an optimization strategy that is implemented during the development of fatigue (11). Consequently, it could occur without any change in the mechanical output or alteration in the maximal performance ( $\mathrm{PI}_{\mathrm{MAX}}$, in this case), but it may still be a limiting factor when exercise is prolonged, probably through the development of dyspnea $(31,22)$. Sufficient data are lacking to venture a hypothesis about whether the adaptation depresses the excitability of the motor command - as reflected by a decrease in MEP amplitude - or whether decreased excitability results in reorganized recruitment. We nevertheless suspect that the second position will be borne out and propose that early in the E + ITL trial, mechanical afferences led to a relative inhibition of the diaphragm motor command that later normalized. Even though it results in dyspnea, which is another constraint, the recruitment of neck and rib cage muscles controls the major stress to the respiratory system, i.e. the inspiratory load. It is possible that in this condition the excitability of the respiratory pathways is reset up during the exercise itself and is not influenced by slighter changes in the homeostasis. Longer inspiratory loaded exercise would be interesting to study to assess whether dyspnea continues to act as a safety feature by leading to the end of exercise before the respiratory system adapts to metabolic changes through variation in the excitability of its command. In contrast, a finer regulation of the diaphragm command, based on both mechanical and chemical afferences, 
could be sought during exercise. This would result in a prolonged relative domination of inhibitory over excitatory mechanisms.

In conclusion and in spite of its limitations, this study seems to contribute to the current body of knowledge suggesting that several mechanisms act in concert to protect the diaphragm against overload-induced damage. These mechanisms include a reduced central activation in loaded conditions (6), the early occurrence of supraspinal fatigue or inhibition (21), and agonist recruitment to overcome an increased demand. 
We are grateful to those who accepted to serve as subjects in this study.

\section{GRANTS}

The Laboratoire des Adaptations Physiologiques aux Activités Physiques received equipment funding (03/RPC-R-034) from the "Région Poitou-Charentes" for this study.

Professor T Similowski was supported in part by a "subvention triennale legs Poix de la Chancellerie de l'Université de Paris, France". 


\section{REFERENCES}

1. Akima, H., J.M. Foley, B.M. Prior, G.A. Dudley, and R.A. Meyer. Vastus lateralis fatigue alters recruitment of musculus quadriceps femoris in humans. J Appl Physiol 92: 679-84, 2002.

2. Aliverti, A., S.J. Cala, R. Duranti, G. Ferrigno, C.M. Kenyion, A. Pedotti, G. Scano, P. Sliwinski, P.T. Macklem, and S. Yan. Human respiratory muscle actions and control during exercise. J Appl Physiol 83: 1256-69, 1997.

3. Balbi, P., A. Perretti, M. Sannino, L. Marcantonio, and L. Santoro. Postexercise facilitation of motor evoked potentials following transcranial magnetic stimulation: a study of normal subjects. Muscle Nerve 25: 448-52, 2002.

4. Barton, P.M., K.C. Hayes. Neck flexor muscle strength, efficiency, and relaxation times in normal subjects and subjects with unilateral neck pain and headache. Arch Phys Med Rehabil 77: 680-7, 1996.

5. Bellemare, F. and A. Grassino. Effect of pressure and timing of contraction on human diaphragm fatigue. J Appl Physiol 53(5): 1190-5, 1982.

6. Bellemare, F. and B. Bigland-Ritchie. Central components of diaphragmatic fatigue assessed by phrenic nerve stimulation. J Appl Physiol 62: 1307-16, 1987.

7. Bigland-Ritchie, B., D.A. Jones, G.P. Hosking, and R.H. Edwards. Central and peripheral fatigue in sustained maximum voluntary contractions of human quadriceps muscle. Clin Sci Mol Med 54: 609-12, 1978.

8. Brasil-Neto, J. P., A. Pascual-Leone, J. Valls-Sole, A. Cammarota, and L. H. M. Cohen. Postexercise depression of motor evoked potentials: a measure of central nervous system fatigue. Exp Brain Res 93: 181-4, 1993.

9. Brasil-Neto, J.P., L.G. Cohen, and M. Hallett. Central fatigue as revealed by postexercise decrement of motor evoked potentials. Muscle Nerve 17: 713-719, 1994.

10. Campbell, E.J.M. An electromyographic study of the role of the abdominal muscles in breathing. J Physiol (London) 117: 222-33, 1952.

11. Eastwood, P.R., D.R. Hillman, and K.E. Finucane. Ventilatory responses to inspiratory threshold loading and role of muscle fatigue in task failure. J Appl Physiol 76: 185-95, 1994.

12. Fitting, J.W., T.D. Bradley, P.A. Easton, M.J. Lincoln, M.D. Goldman, and A. Grassino. Dissociation between diaphragmatic and rib cage muscle fatigue. $J$ Appl 
Physiol 64: 959-65, 1988.

13. Gandevia, S.C. Spinal and supra-spinal factors in human muscle fatigue. Physiol rev 81: 1725-90, 2001.

14. Guleria, R., R. Lyall, N. Hart, M.L. Harris, C. H. Hamnegård, M. Green, J. Moxham, and M. I. Polkey. Central fatigue of the diaphragm and quadriceps during incremental loading. Lung 180: 1-13, 2002.

15. Hart, N., A.H. Nickol, D. Cramer, S.P. Ward, F. Lofaso, and N.B. Pride. Effect of severe isolated unilateral and bilateral diaphragm weakness on exercise performance. Am J Respir Crit Care Med 165: 1265-70, 2002.

16. Hershenson, M.B., Y. Kikushi, G.E. Tzelepis, and F.D. McCool. Preferential fatigue of the rib cage muscles during inspiratory resistive loaded ventilation. J Appl Physiol 66: 750-4, 1989.

17. Hill, N.S., C. Jacoby, and H.W. Farber. Effect of an endurance triathlon on pulmonary function. Med Sci Sports Exerc 23: 1260-4, 1991.

18. Höllge, J., M. Kunkel, U. Ziemann, F. Tergau, R. Geese, and C.D. Reimers. Central fatigue in sports and daily exercises. A magnetic stimulation study. Int J Sports Med 18: 614-617, 1997.

19. Johnson, B.D., M.A. Babcock, O.E. Suman, and J.A. Dempsey. Exercise-induced diaphragmatic fatigue in healthy humans. J Physiol (London) 460: 385-405, 1993.

20. Loke, J., D.A. Mahler, and J.A. Virgulto. Respiratory muscle fatigue after marathon running. J Appl Physiol 52: 821-4, 1982.

21. Macklem, P.T., D.M. Macklem, A. De Troyer. A model of inspiratory muscle mechanics. J Appl Physiol 55: 547-57, 1983.

22. Markov, G., C.M. Spengler, C. Knopli-Lenzin, C. Stuessi, and U. Boutellier. Respiratory muscle training increases cycling endurance without affecting cardiovascular responses to exercise. Eur J Appl Physiol 85: 233-9, 2001.

23. McKenzie, D.K., B. Bigland-Ritchie, R.B. Gorman, and S.C. Gandevia. Central and peripheral fatigue of human diaphragm and limb muscles assessed by twitch interpolation. J Physiol (London) 454: 643-56, 1992.

24. Merton, P.A. Voluntary strength and fatigue. J Physiol (London) 123: 553-64, 1954.

25. Polkey, M.I., D. Kyroussis, C.-H. Hamnegard, G.-H. Mills, M. Grenn, and J. Moxham. Quadriceps strength and fatigue assessed by magnetic stimulation of the femoral nerve in man. Muscle Nerve 19: 549-55, 1996.

26. Romagnoli, I., F. Gigliotti, B. Lanini, R. Bianchi, N. Soldani, M. Nerini, R. Duranti, 
and G. Scano. Chest wall kinematics and respiratory muscle coordinated action during hypercapnia in healthy males. Eur J Appl Physiol 91: 525-33, 2004.

27. Sacco, P., G.W. Thickbroom, M.L. Byrnes, and F.L. Mastaglia. Changes in corticomotor excitability after fatiguing muscle contractions. Muscle Nerve 23: 1840-6, 2000.

28. Samii, A., E.M. Wasserman, K. Ikoma, B. Mercuri, and M. Hallet. Characterization of postexercise facilitation and depression of motor evoked potentials to transcranial magnetic stimulation. Neurology 46: 1376-82, 1996.

29. Similowski, T., C. Straus, L. Coïc, and J.-P. Derenne. Facilitation-independent response of the diaphragm to cortical magnetic stimulation. Am J Respir Crit Care Med 154: 1771-7, 1996.

30. Sliwinski, P., S. Yan, A.P. Gauthier, and P.T. Macklem. Influence of global inspiratory muscle fatigue on breathing during exercise. J Appl Physiol 80: 1270-8, 1996.

31. Spengler, C.M., and U. Boutellier. Breathless legs? Consider training your respiration. News Physiol Sci, 15: 101-5, 2000.

32. Taylor, J.L., J.E. Butler, G.M. Allen, and S.C. Gandevia. Changes in motor cortical excitability during human muscle fatigue. 490: 519-28, 1996.

33. Tergau, F., R. Geese, A. Bauer, S. Baur, W. Paulus, and C. Detlev Reimer. Motor cortex fatigue in sports measured by transcranial magnetic double stimulation. Med Sci Sports Exerc 32: 1942-8, 2000.

34. Vérin, E., C. Straus, A. Demoule, P. Mialon, J.-P. Derenne, and T. Similowski. Measurement of phrenic nerve conduction time from surface electrodes in man: validation of improved electrode placement with needle diaphragm recordings. J Appl Physiol 62: 967-74, 2002.

35. Vérin, E., E. Ross, A. Demoule, N. Hopkinson, A. Nickol, B. Fauroux, J. Moxham, T. Similowski, and M.I. Polkey. Effects of exhaustive incremental treadmill exercise on diaphragm and quadriceps motor potentials evoked by transcranial magnetic stimulation. J Appl Physiol 96: 253-9, 2004.

36. Ward, M.E., D. Eidelman, D.G. Stubbing, F. Bellemare, and P.T. Macklem. Respiratory sensation and pattern of respiratory muscle activation during diaphragm fatigue. J Appl Physiol 65: 2181-9, 1988.

37. Wasserman, K., B. J. Whipp, S. N. Koyal, and W. L. Beaver. Anaerobic threshold and respiratory gas exchange during exercise. J Appl Physiol 35: 236-42, 1973.

38. Yan, S., P. Sliwinski, A. P. Gauthier, I. Lichros, S. Zakynthinos, and P.T. Macklem. Effect of global inspiratory muscle fatigue on ventilatory and respiratory muscle 
responses to $\mathrm{CO}_{2}$. J Appl Physiol 75: 1371-7, 1993.

39. Zocchi, L., J.W. Fitting, M.C. Fracchia, C. Rampulla, and A. Grassino. Effect of pressure and timing of contraction on human rib cage muscle fatigue. Am Rev Respir Dis 147: 857-64, 1993. 
Table 1. Individual characteristics of the subjects. MAP: maximal aerobic power, defined as the highest power the subject was able to maintain for one minute during the incremental test. In the gender section, $\mathrm{m}$ stands for male and $\mathrm{f}$ for female.

Figure 1. Individual and mean variations between the two sessions: exercise (E) and inspiratory-loaded exercise (E + ITL), for the following ventilatory parameters: A) ventilatory output, B) breathing frequency, C) tidal volume, D) respiratory duty cycle, i.e. ratio between inspiratory time and total time of a cycle. NS: non-significant.

Figure 2. Individual and mean $( \pm \mathrm{SD})$ variations between the two sessions: exercise $(\mathrm{E})$ and inspiratory-loaded exercise (E + ITL), for A) dyspnea rated on a Borg scale and B) mean heart rate. $*: p<0.05 ; * *: p<0.01$.

Figure 3. A) Mean and SD integrated electromyographic activity (EMGi) of the sternocleidomastoid muscles over the time of $\mathrm{E}(\square)$ and $\left.\mathrm{E}+\operatorname{ITL}(\boldsymbol{\square}) . \mathrm{n}=6 .{ }^{* *}: p<0.01 . \mathrm{B}\right)$ Mean and SD amplitude of the average deflection of Pdi during the sessions; no statistical analysis was performed on these data since the number of subjects is small $(n=3)$.

Figure 4. Representative pressure traces recorded in one subject at the beginning of $\mathrm{E}$ (A) and during a corresponding period in E + ITL (B). The transdiaphragmatic pressure is in grey, gastric pressure is represented by the black trace in phase with Pdi, and oesophageal pressure is the remaining black trace. The electromyographic activity of the sternocleidomastoid is displayed below the pressure traces. 
Figure 5. A) Mean and SEM for PI MAX before (rest) and 30 minutes (P30) after the end of exercise without ( $\square=$ exercise trial) and with $(\boldsymbol{\square}=$ inspiratory loaded exercise trial) an inspiratory load. B) Mean and SEM for mouth pressure in response to cervical magnetic stimulation before (rest), and 15 (P15) and 25 minutes (P25) after the end of the trials. $\mathrm{n}=6$.

The interaction effect was non-significant (NS, $p=0.96$ and 0.71 in $\mathrm{A}$ and $\mathrm{B}$, respectively).

Figure 6. Representative M-wave trace recorded in one subject at the measurement time points of baseline and 15 and 25 minutes post-exercise (post $15 \mathrm{~min}$ and post $25 \mathrm{~min}$, respectively) of the exercise trials (A) and the inspiratory loaded exercise trials (B).

Figure 7. Mean and SEM for MEP amplitudes of the diaphragm (A) and rectus femoris (B) at rest, and 10 (P10), 20 (P20) and 40 (P40) minutes after the end of exercise. $\square$ exercise (no

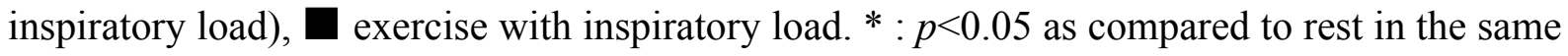
condition; \# : $p<0.05$ as compared to the other condition at the same time. NS: non-significant interaction effect between the time and condition effects. 


\begin{tabular}{|c|c|c|c|c|c|c|c|}
\hline Subject & $\begin{array}{c}\text { Gender } \\
\mathrm{m} / \mathrm{f}\end{array}$ & $\begin{array}{c}\text { Age } \\
\text { (years) }\end{array}$ & $\begin{array}{c}\text { Height } \\
\text { (cm) }\end{array}$ & $\begin{array}{c}\text { Weight } \\
(\mathrm{kg})\end{array}$ & $\begin{array}{c}\text { MAP } \\
(\mathrm{W})\end{array}$ & $\begin{array}{l}\dot{\mathrm{V}} \mathbf{O}_{2 \mathrm{MAX}} \\
\left(\mathrm{ml}^{-1} \cdot \mathrm{min}^{-}\right. \\
\left.\quad{ }^{-} \cdot \mathrm{kg}^{-1}\right)\end{array}$ & $\underset{\left(\mathrm{cmH}_{2} \mathrm{O}\right)}{\mathbf{P I}_{\text {MAX }}}$ \\
\hline 1 & $\mathrm{f}$ & 30 & 168 & 65 & 225 & 38.0 & 135 \\
\hline 2 & $\mathrm{~m}$ & 30 & 175 & 79 & 350 & 56.9 & 146 \\
\hline 3 & $\mathrm{f}$ & 33 & 169 & 59 & 175 & 36.0 & 105 \\
\hline 4 & $\mathrm{~m}$ & 24 & 184 & 81 & 300 & 43.5 & 202 \\
\hline 5 & $\mathrm{~m}$ & 25 & 182 & 62 & 290 & 56.3 & 175 \\
\hline 6 & $\mathrm{~m}$ & 27 & 180 & 77 & 350 & 52.0 & 159 \\
\hline 7 & $\mathrm{f}$ & 22 & 170 & 55 & 200 & 36.9 & 100 \\
\hline 8 & $\mathrm{f}$ & 22 & 176 & 66 & 275 & 35.4 & 135 \\
\hline 9 & $\mathrm{f}$ & 26 & 163 & 60 & 200 & 32.0 & 112 \\
\hline 10 & $\mathrm{~m}$ & 21 & 185 & 79 & 350 & 46.6 & 132 \\
\hline Mean & & 26 & 175 & 68 & 276 & 43.4 & 140 \\
\hline SD & & 4 & 7 & 9 & 66 & 9.1 & 32 \\
\hline
\end{tabular}


A

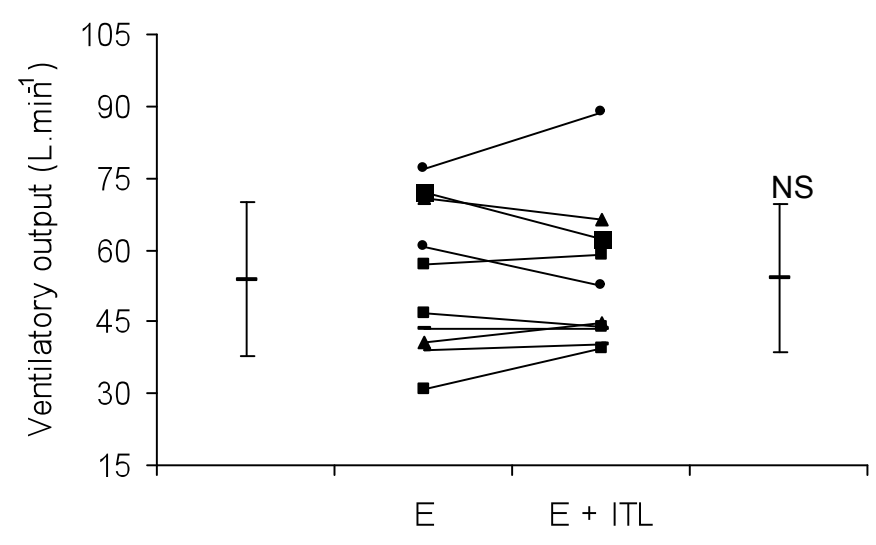

C

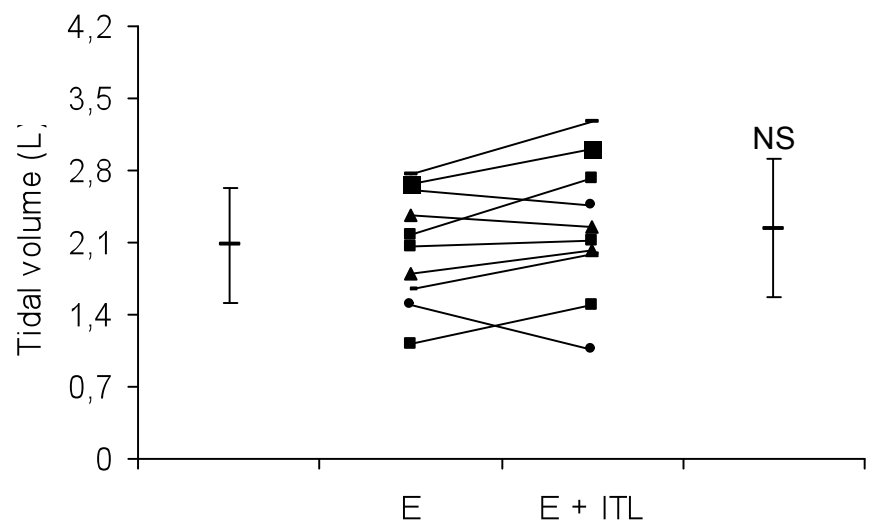

B

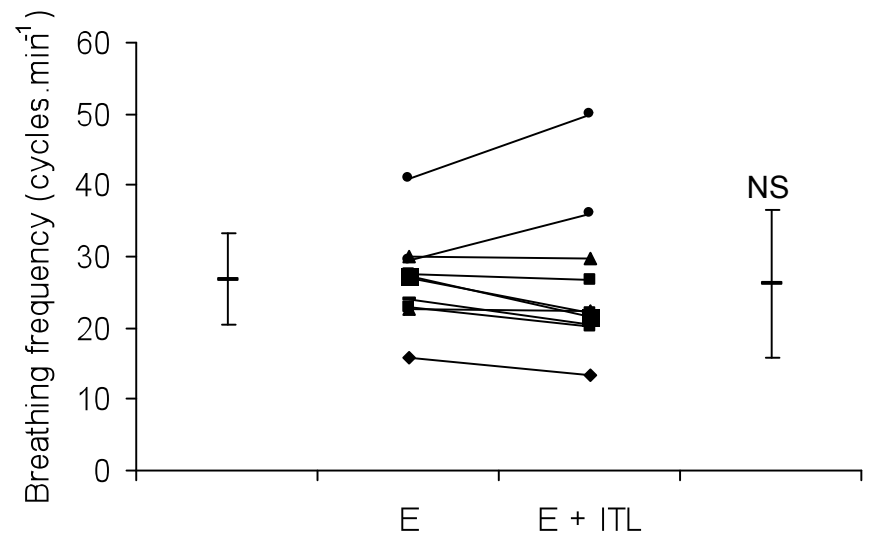

D

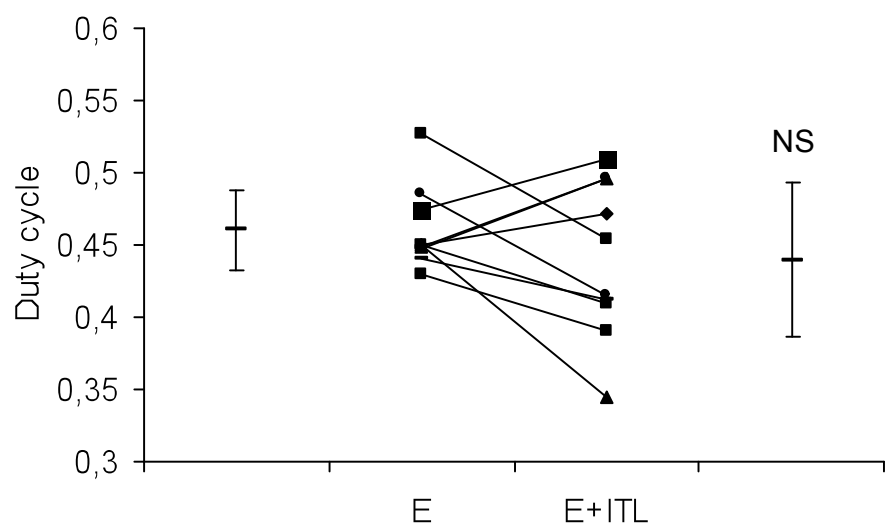


FIGURE 2

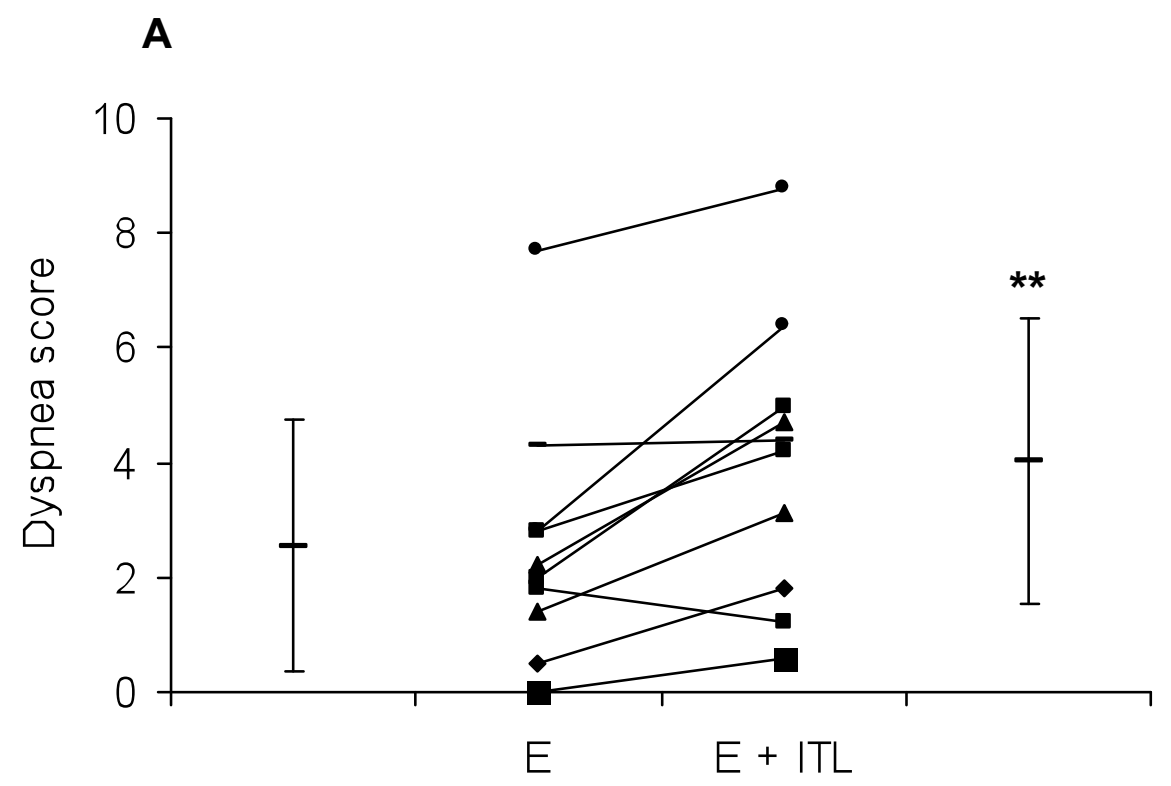

B

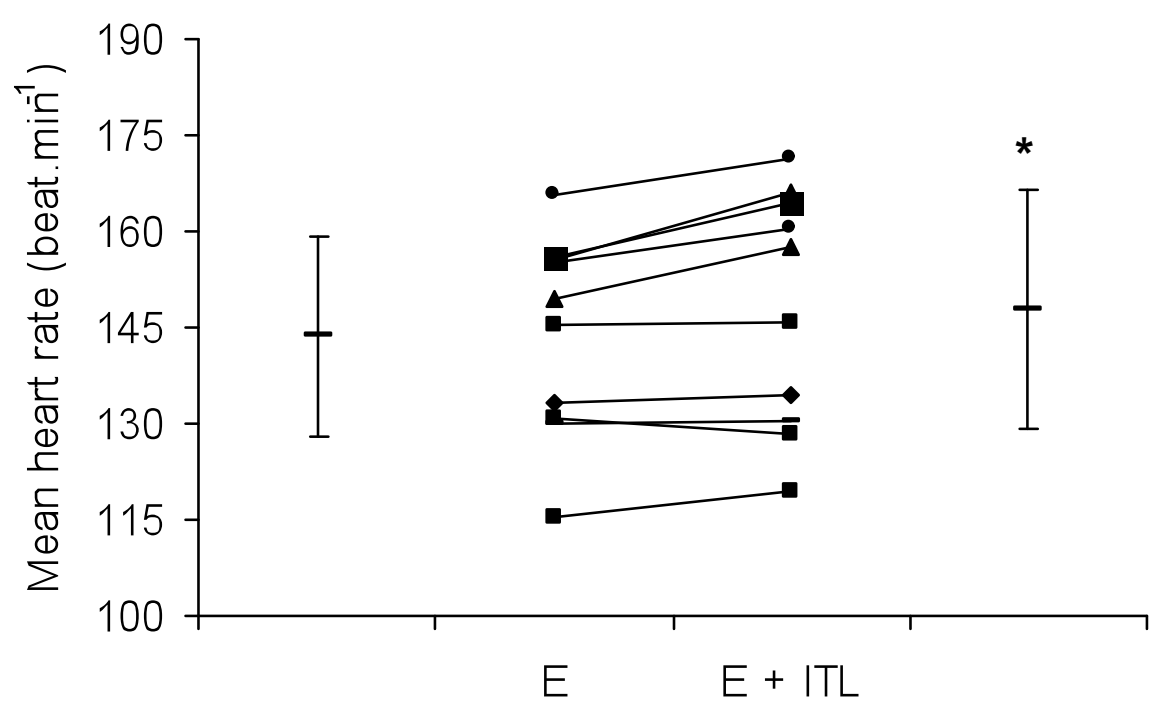


FIGURE 3

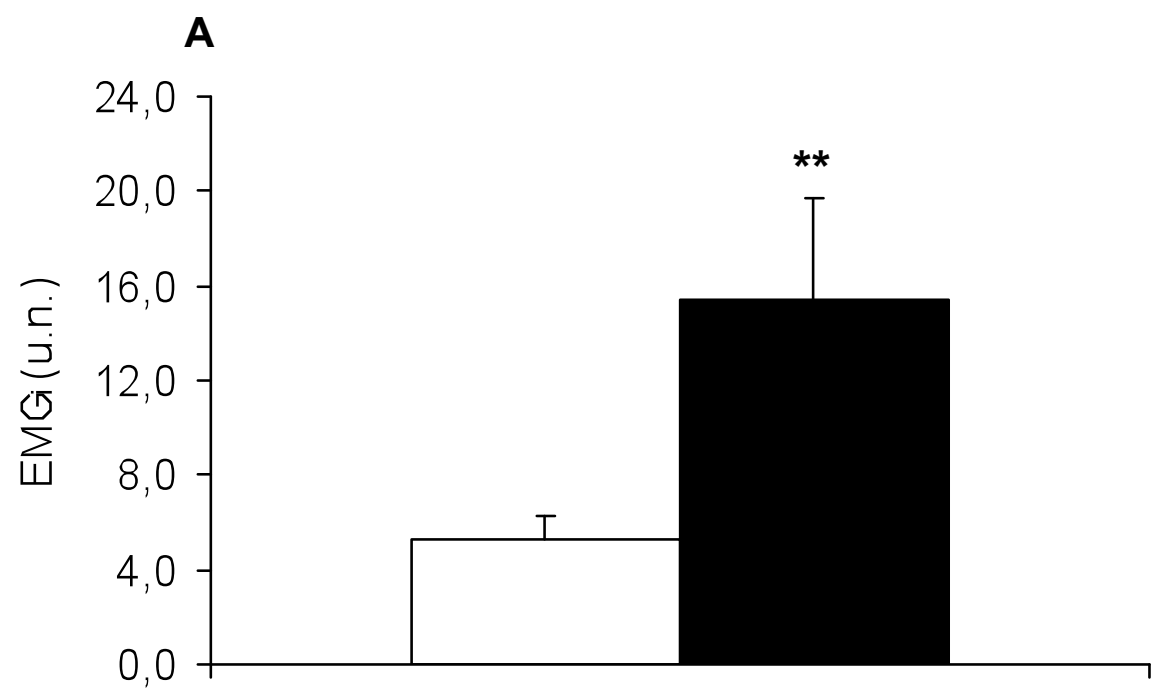

B

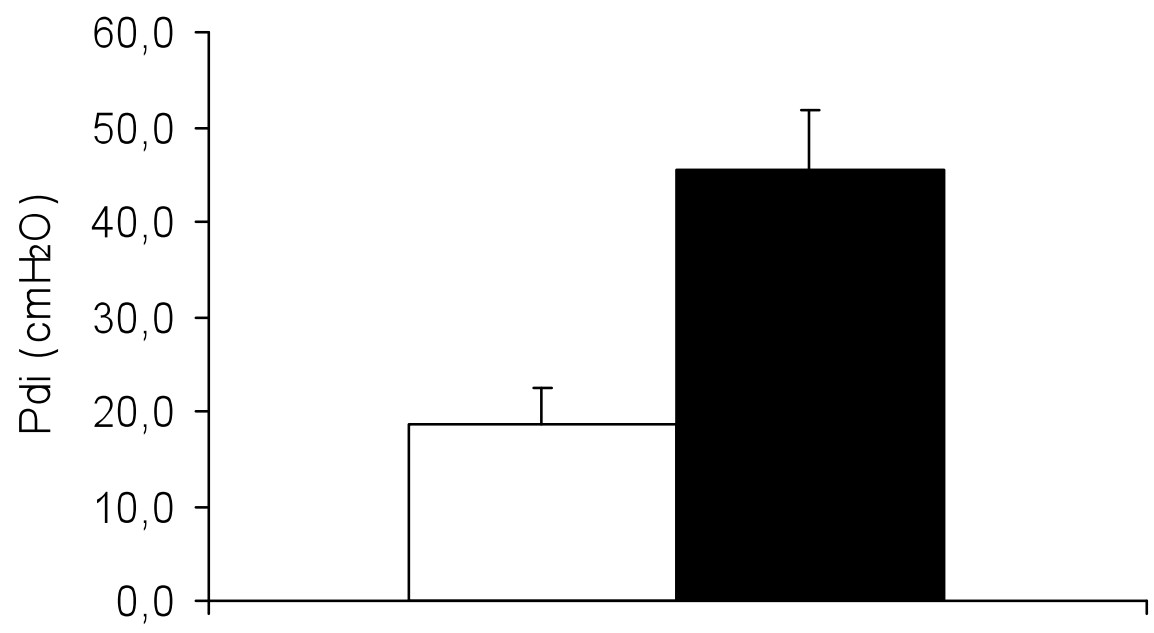


FIGURE 4

A

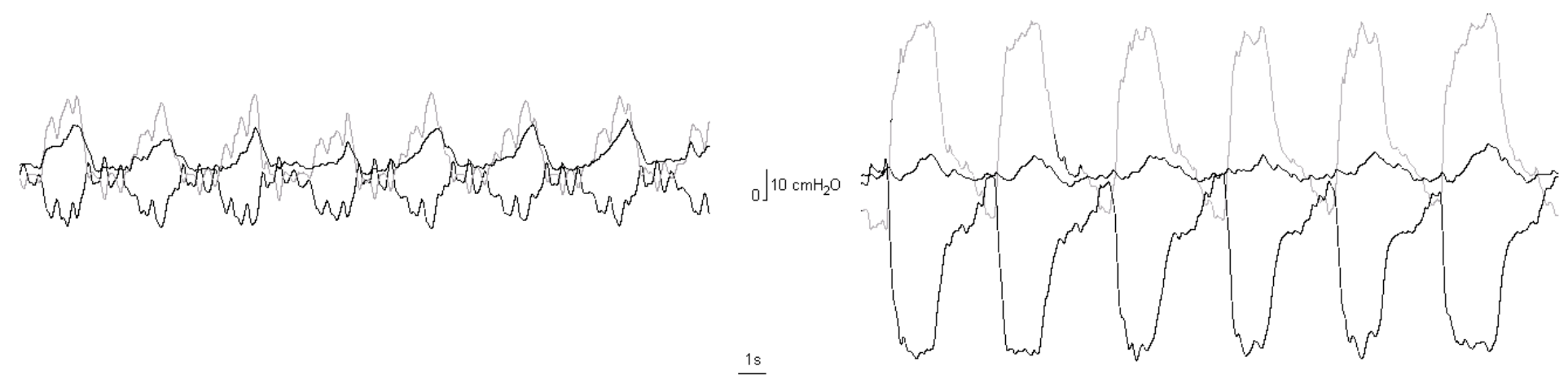

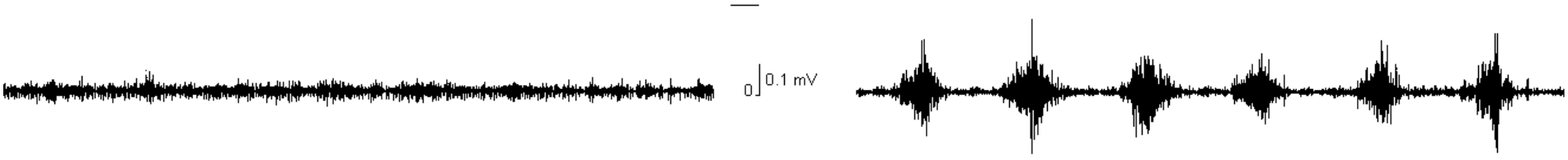



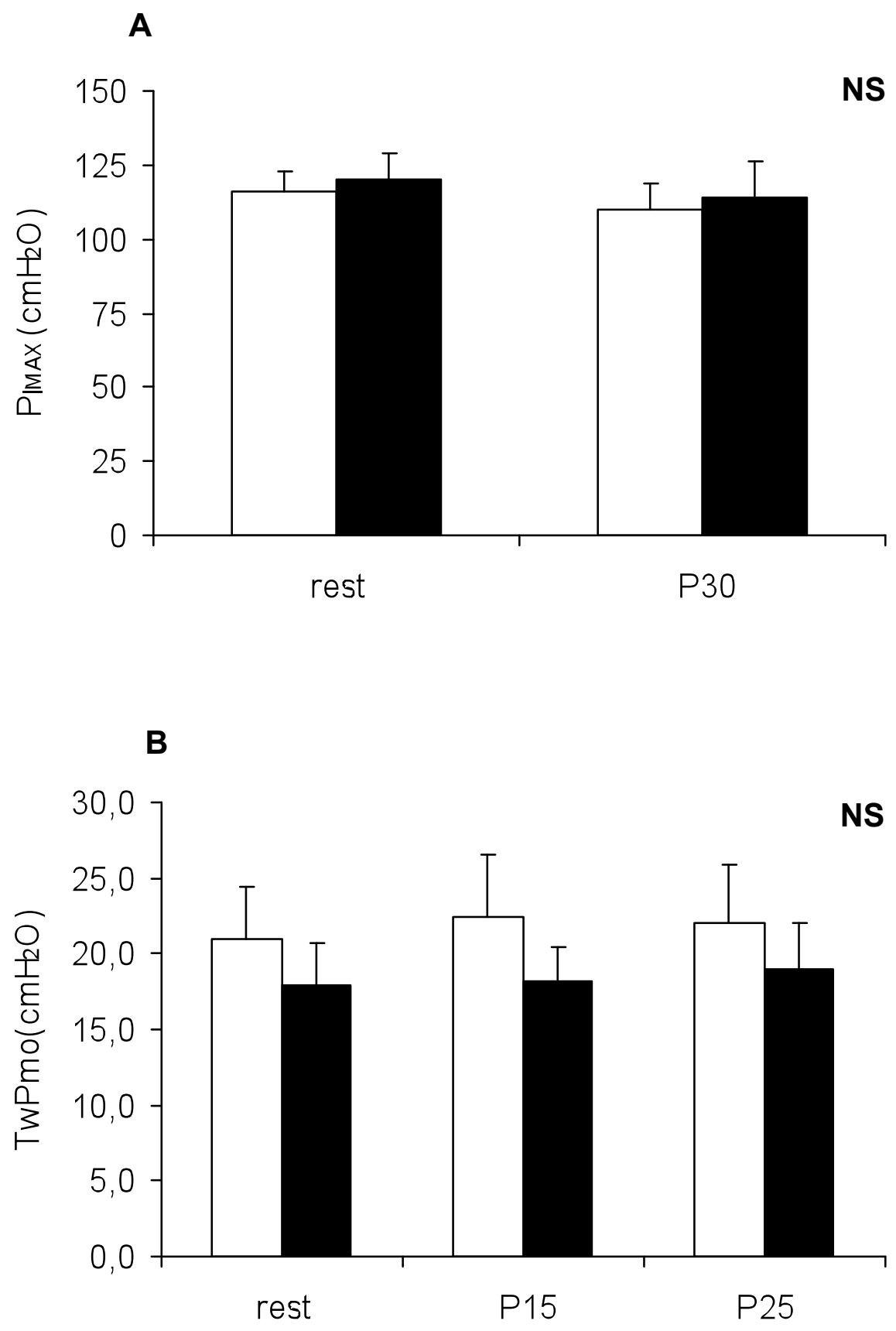


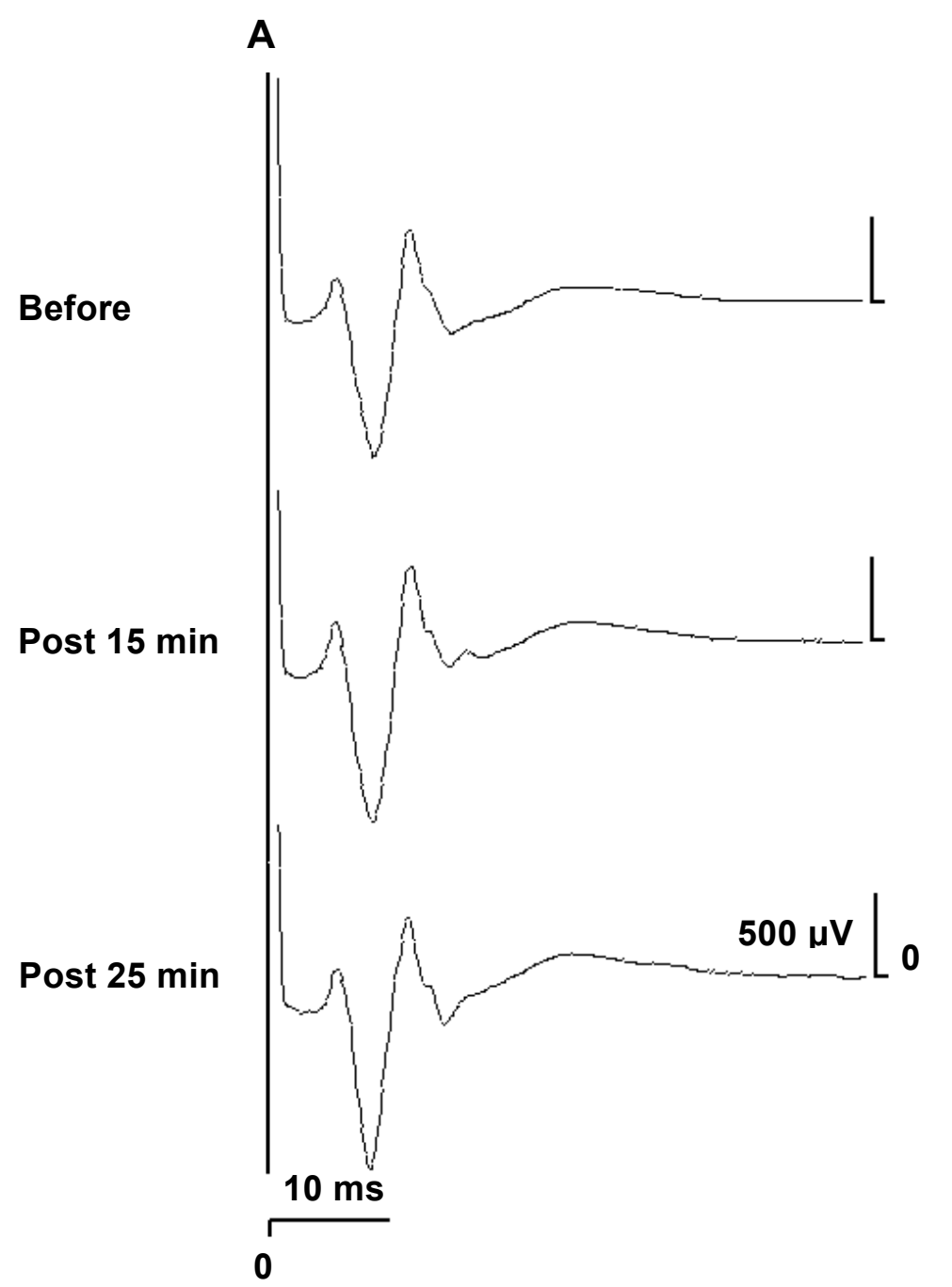

B

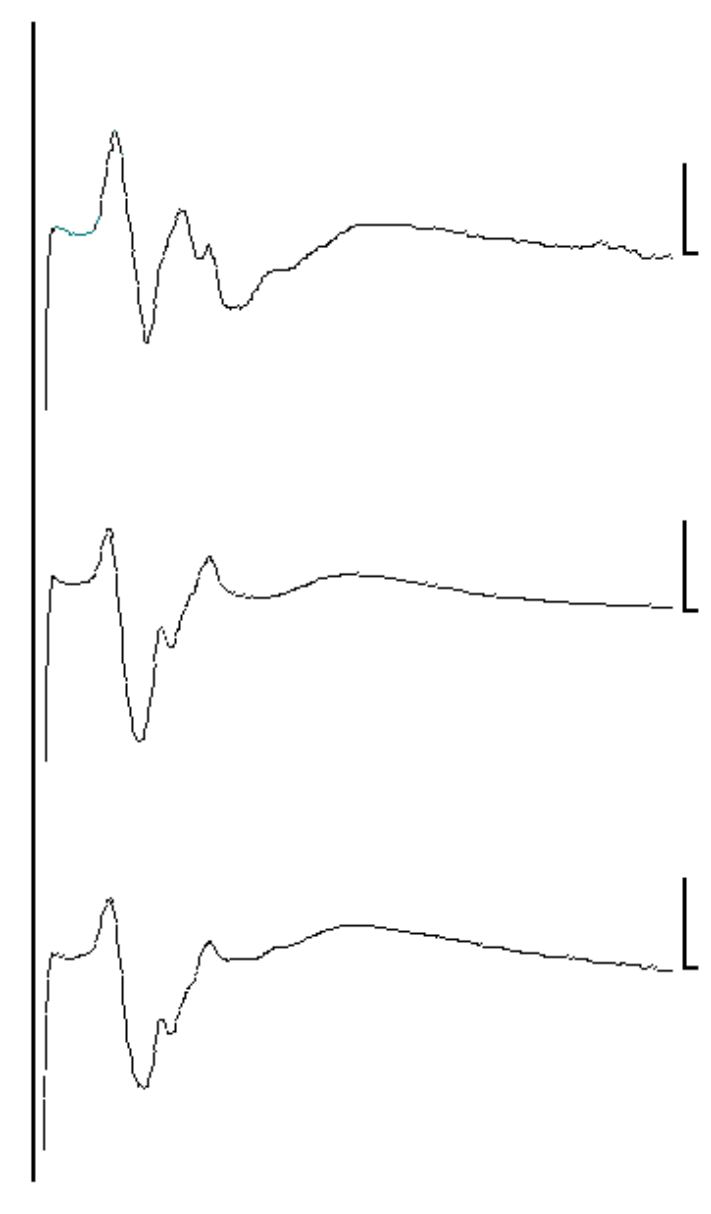



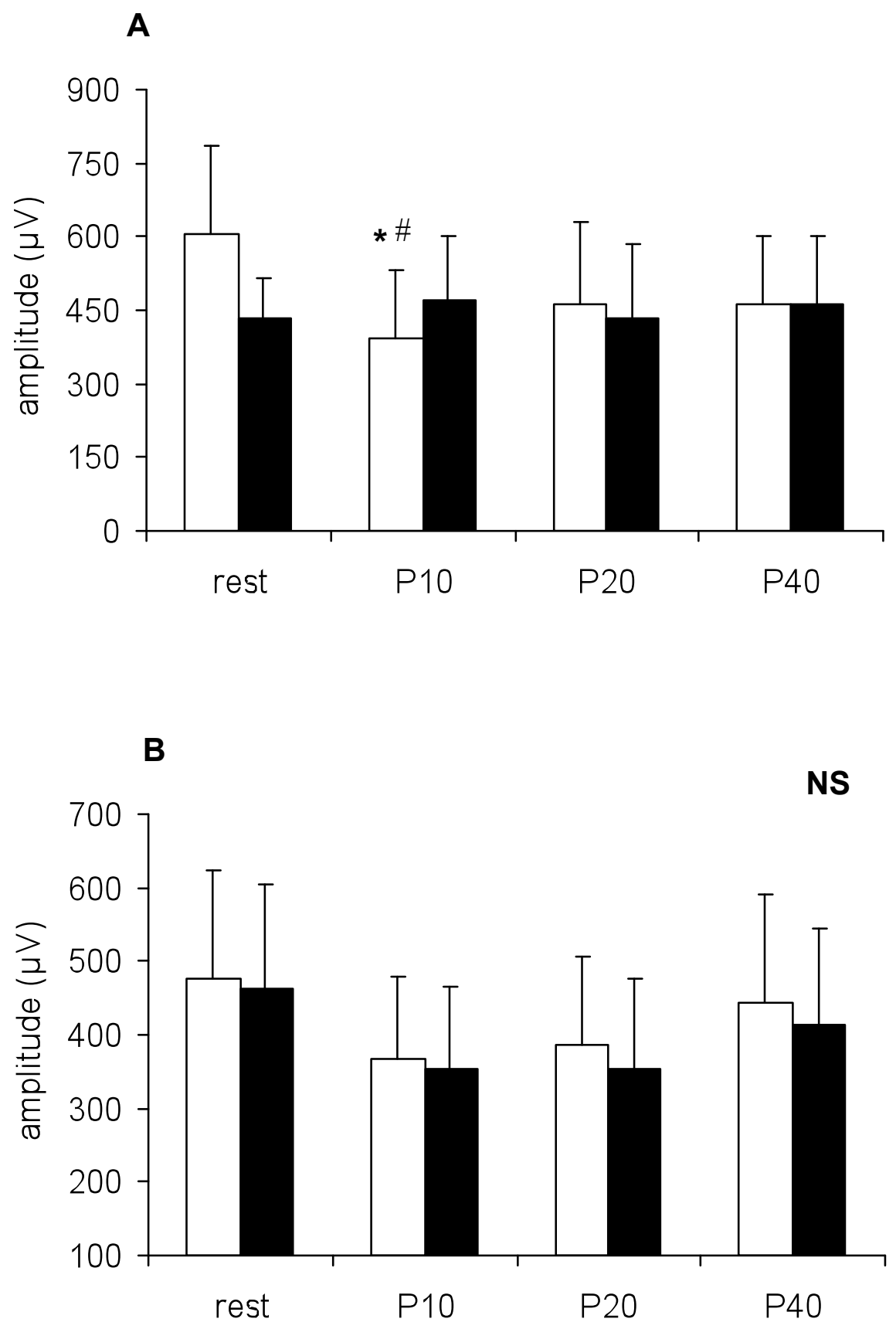